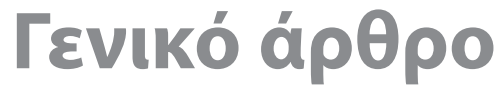 General article
}

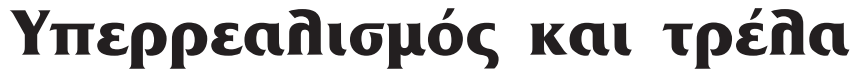

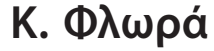

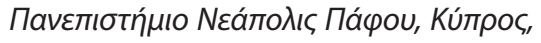

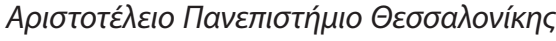

Uuxıatpıkń 2017, 28:360-366

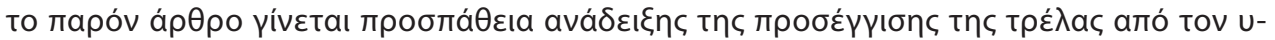

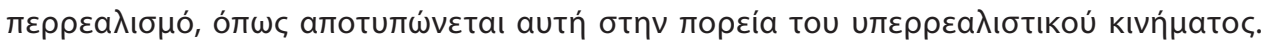

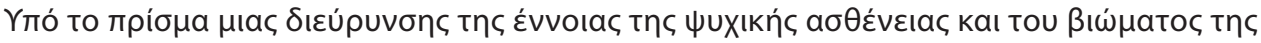

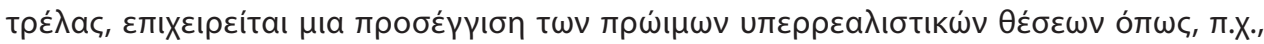

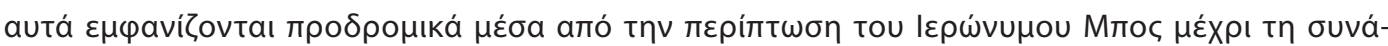

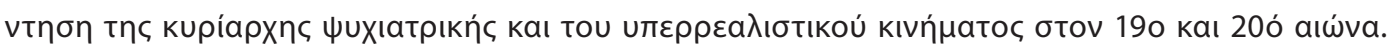

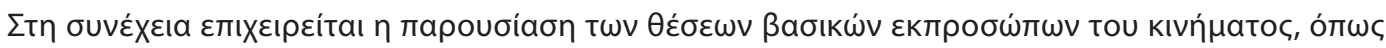

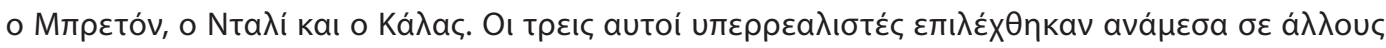

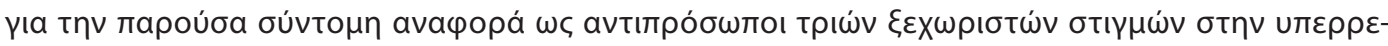

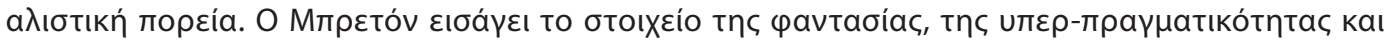

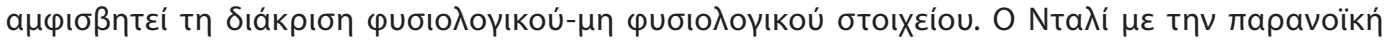

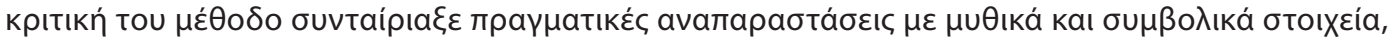

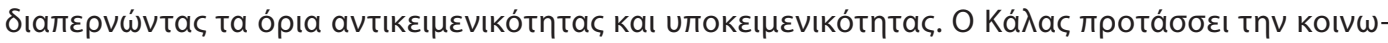

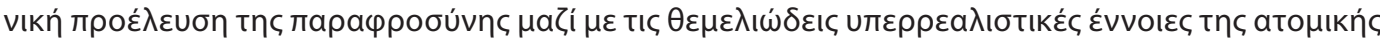

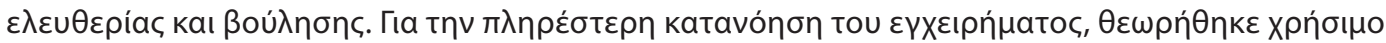

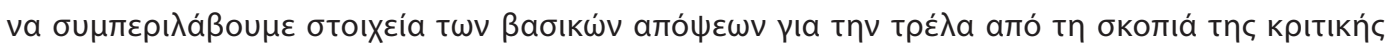

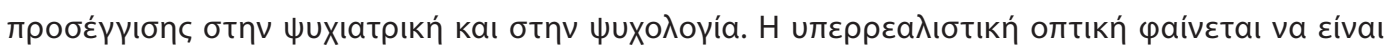

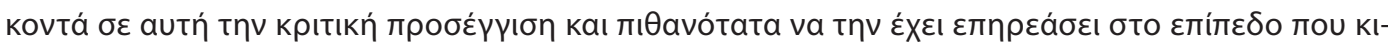

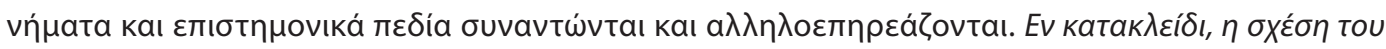

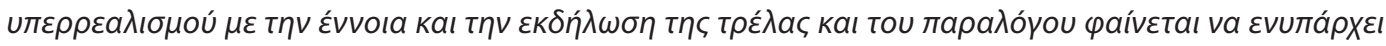

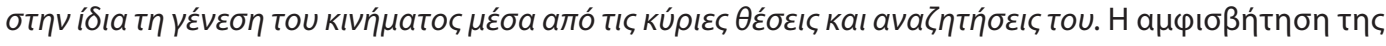
$\lambda \varepsilon \gamma$

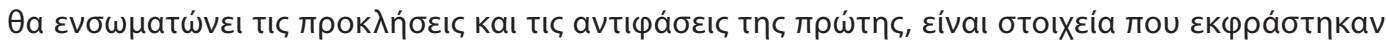

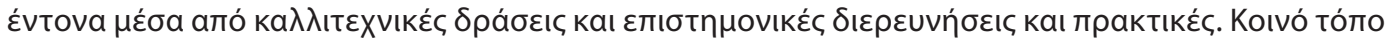

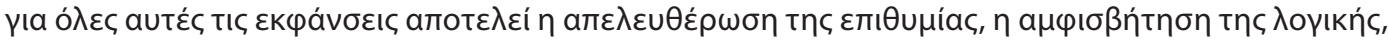




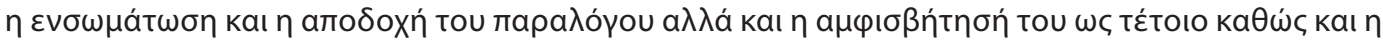

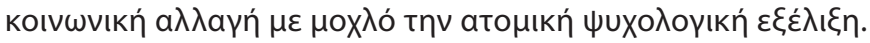

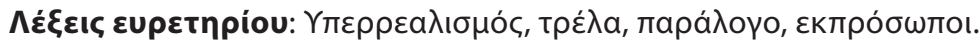

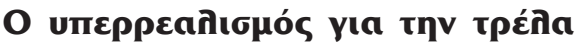 кaı то mapánoyo: 'Eva Xpovıкó}

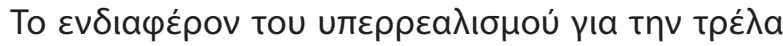

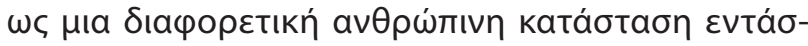

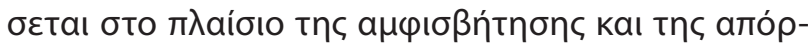

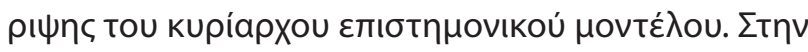

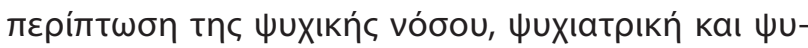

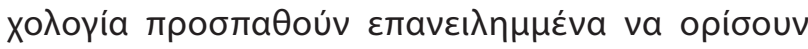

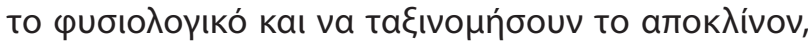

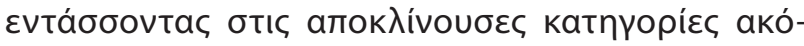

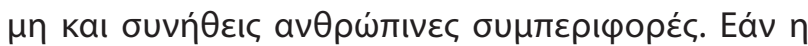

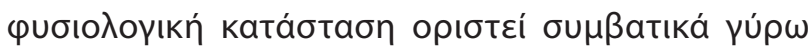

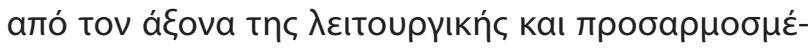

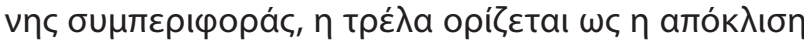

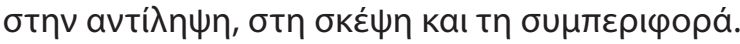

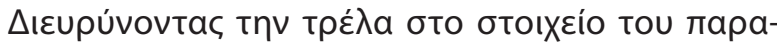

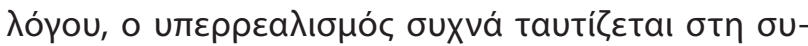

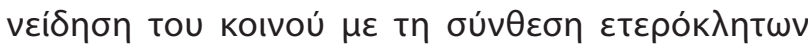

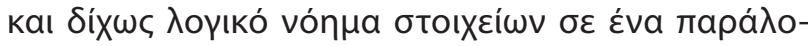

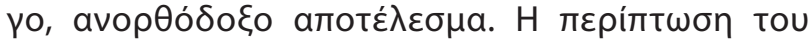

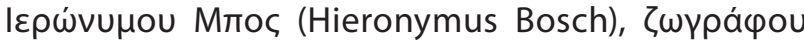

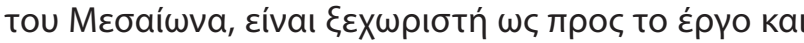

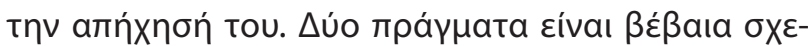

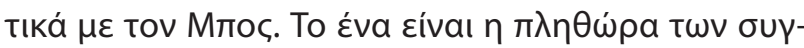

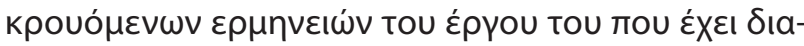

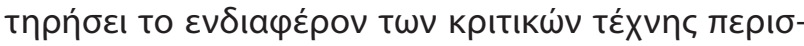

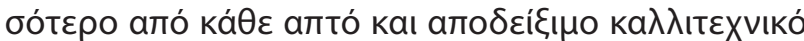

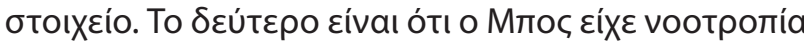

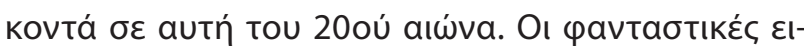

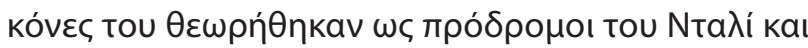

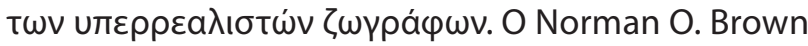

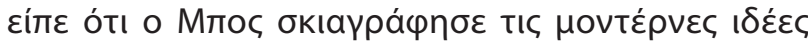

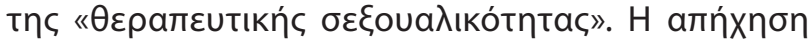

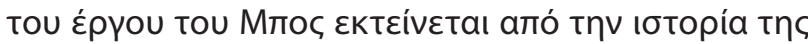

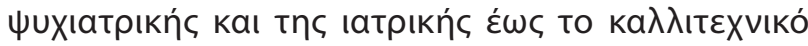

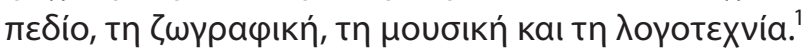

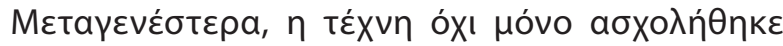

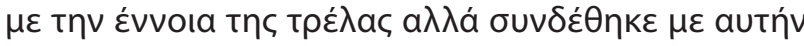
поІкıлотро́тшс. Ато́ то 1809 каı то Illustrations of

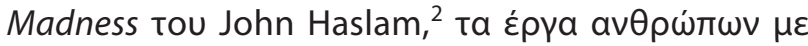

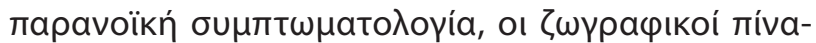

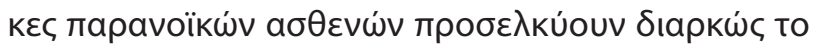

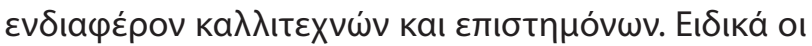

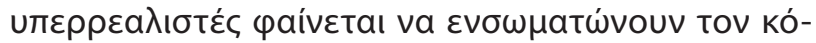

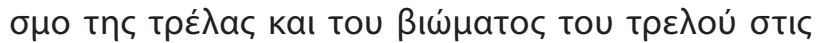

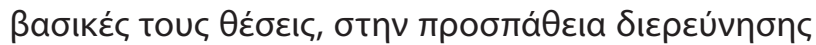

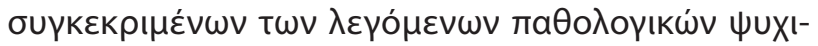

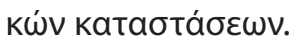

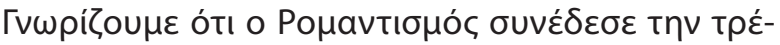

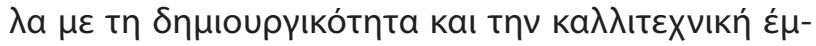

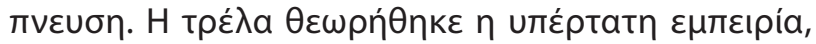

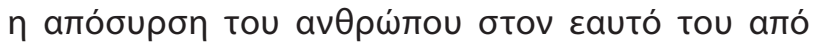

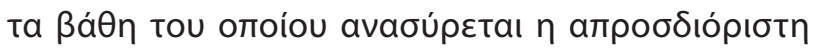

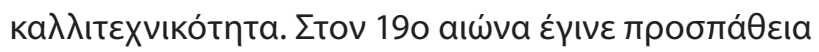

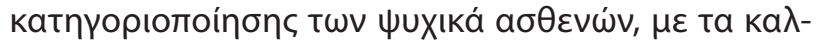

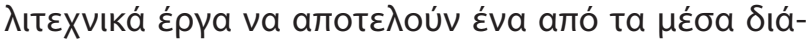

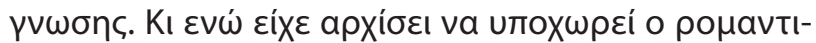

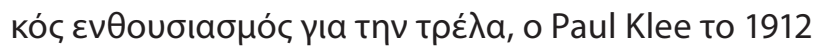

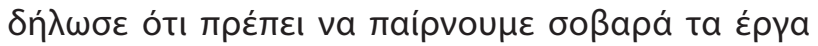

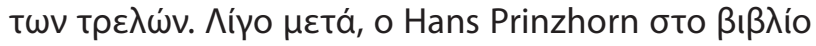

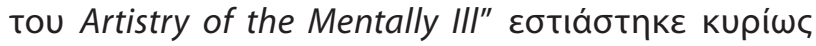

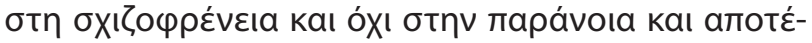

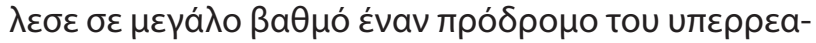

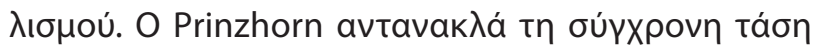

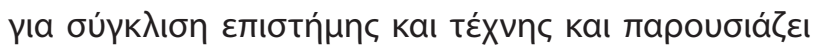

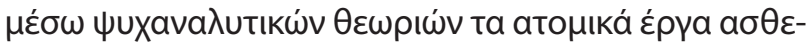

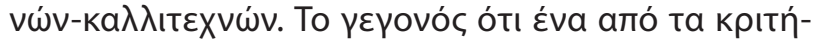

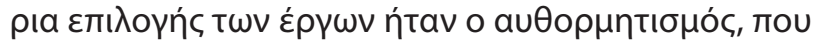

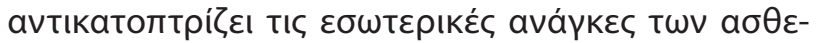

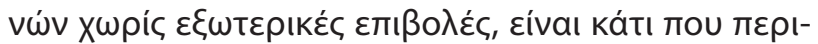

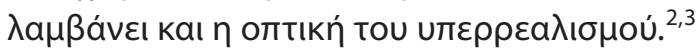

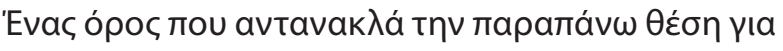

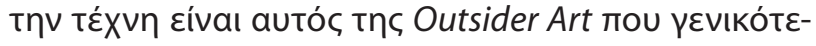

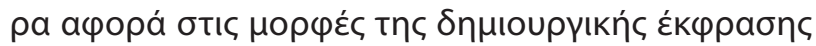

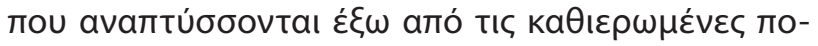

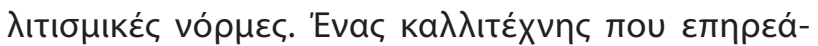

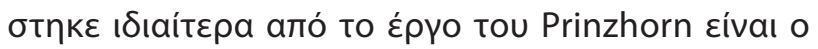

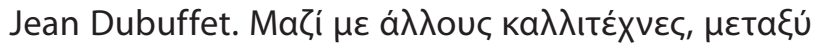

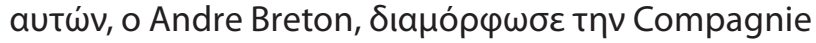

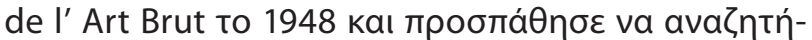

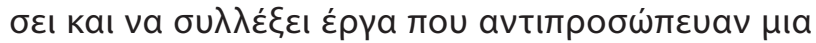




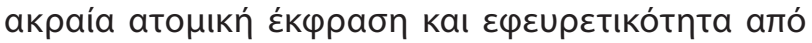

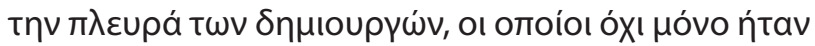

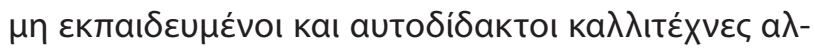

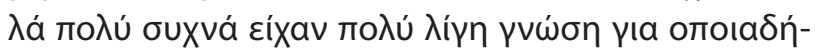

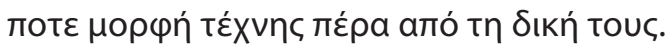

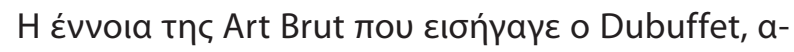

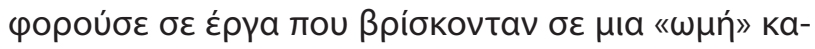

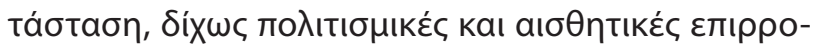

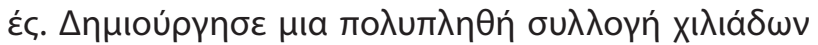

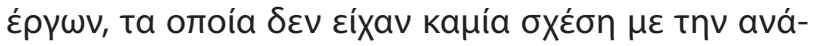

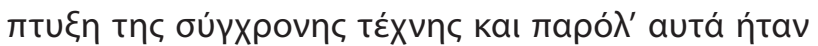

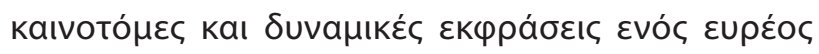

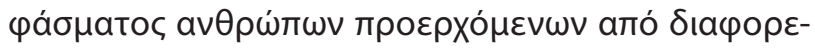
тІко́ ито́ßaӨро.

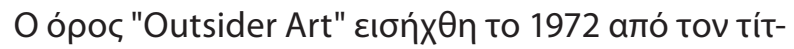

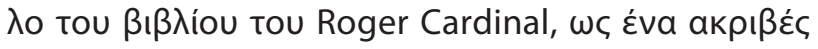

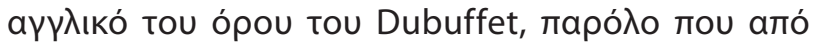

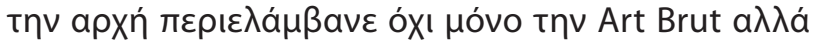

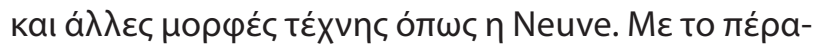

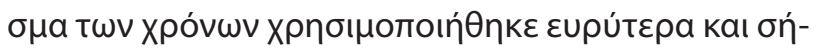

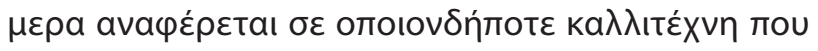

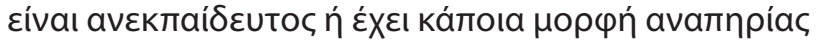

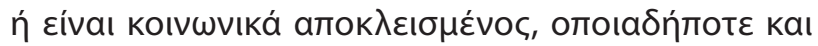

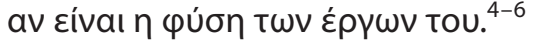

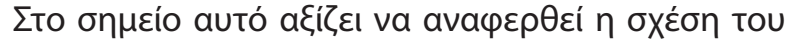

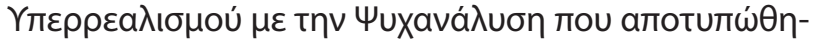

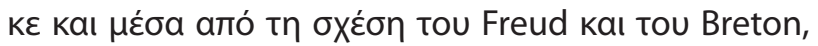

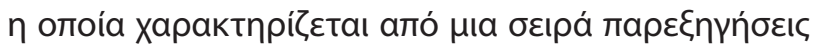

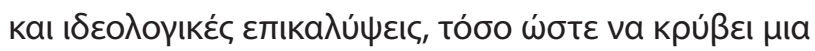

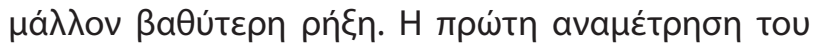

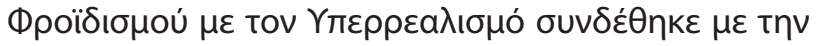

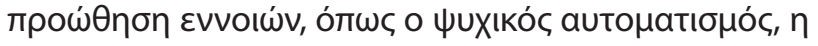

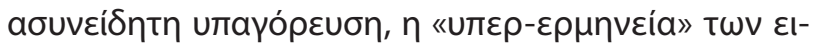

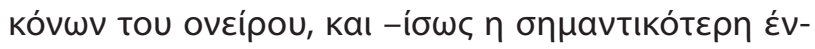

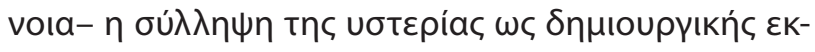

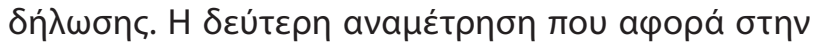

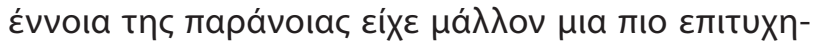

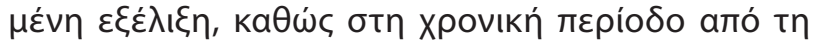

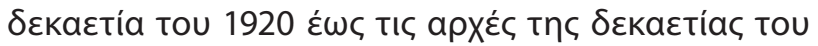

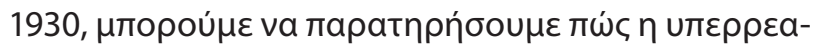

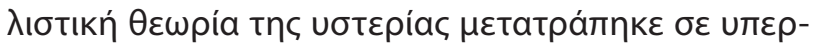

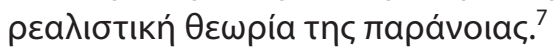

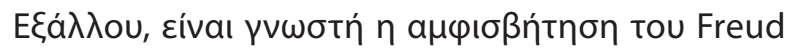

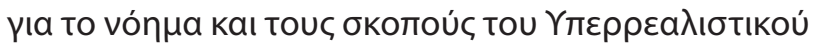

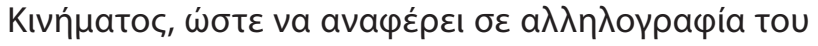

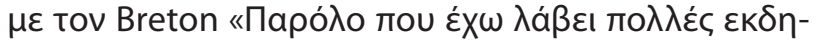

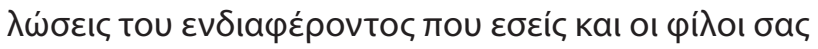

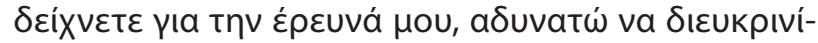

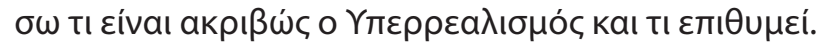

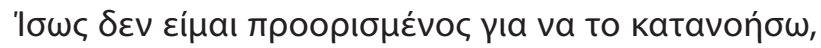

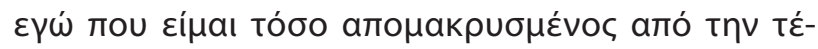

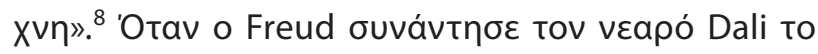

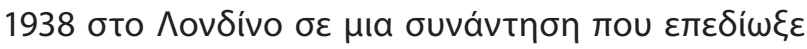

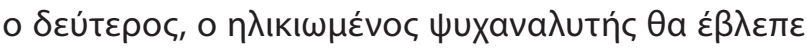

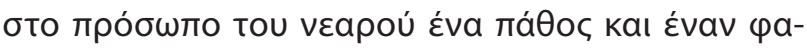

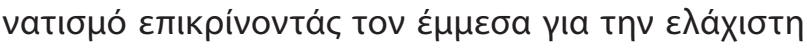

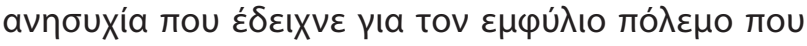

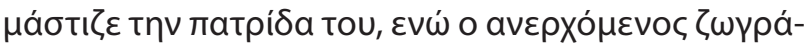

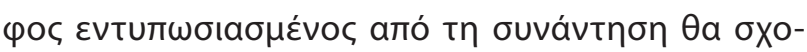

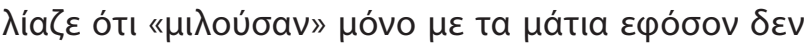

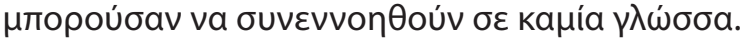

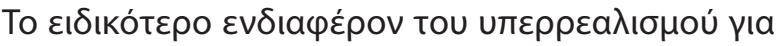

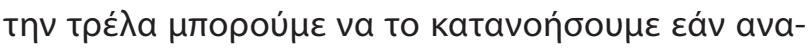

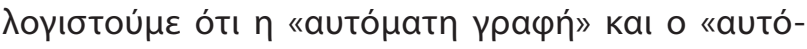

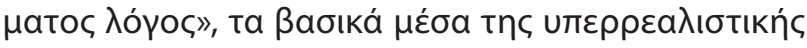

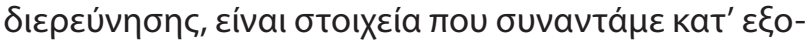

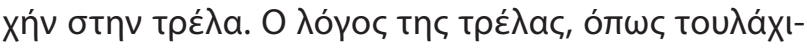

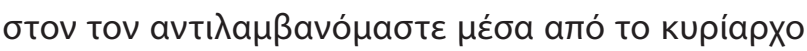

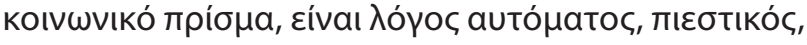

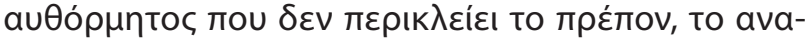

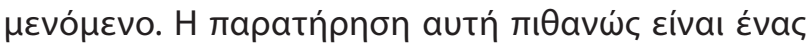

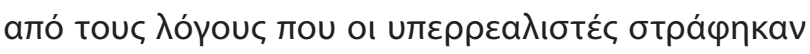

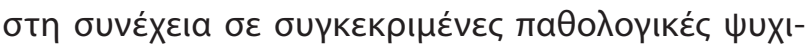

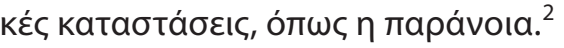

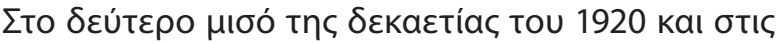

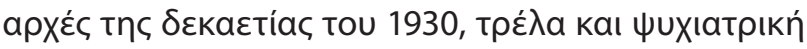

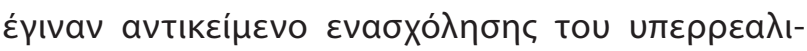

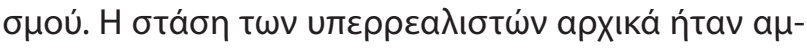

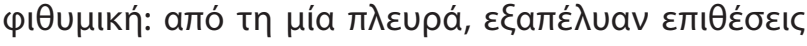

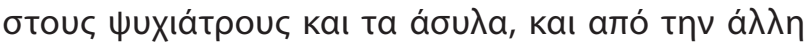

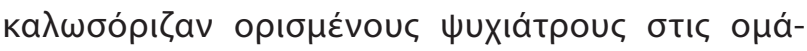

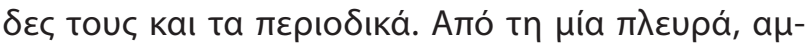

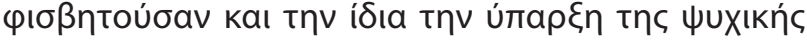

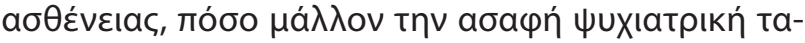

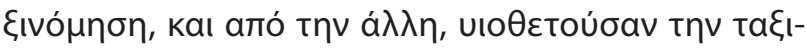

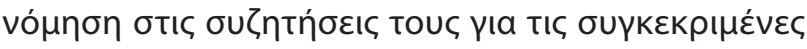

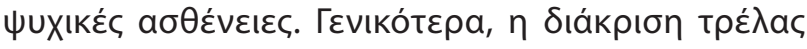

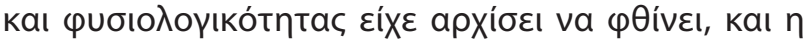

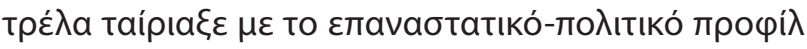

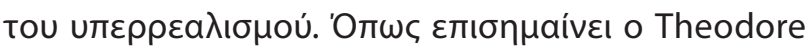
Frankel бто Letter to the Chief Doctors of Asylums, ${ }^{2}$

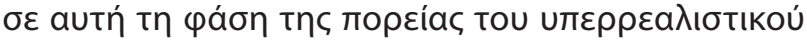

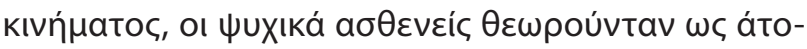




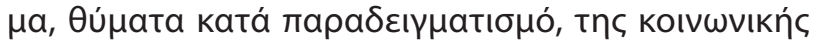
ठıктаторі́ac.

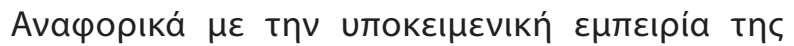

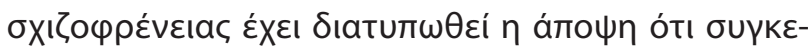

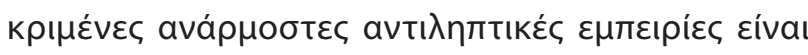

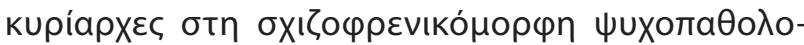

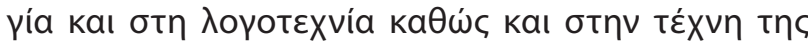

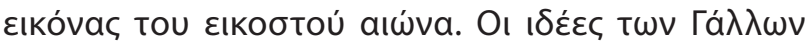

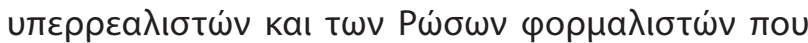

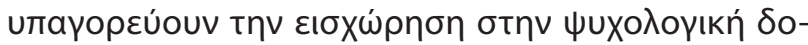

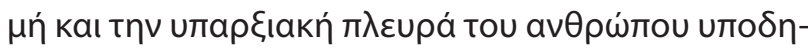

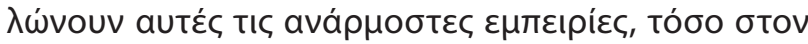

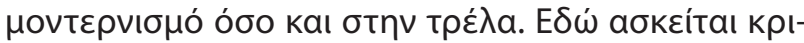

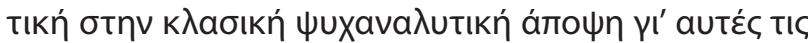

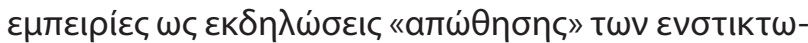

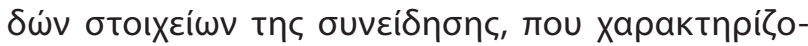

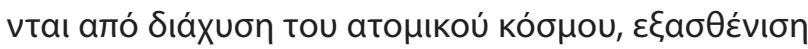

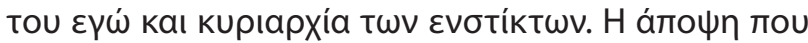

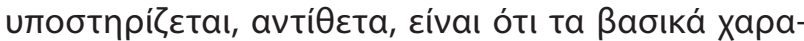

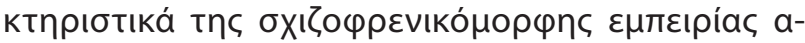

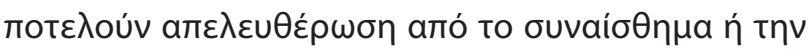

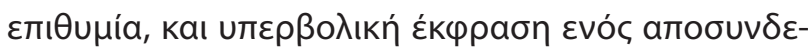

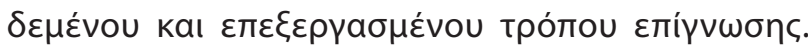

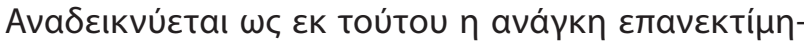

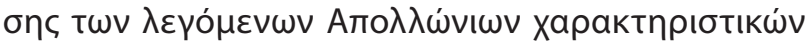

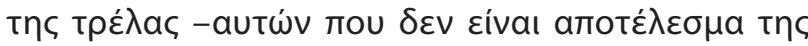

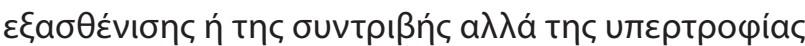

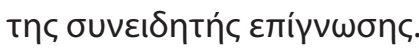

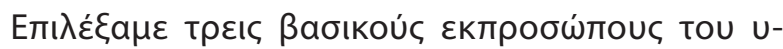

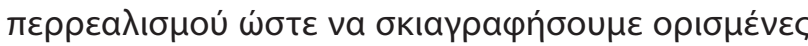

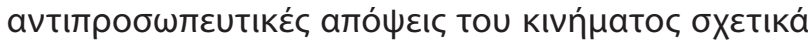

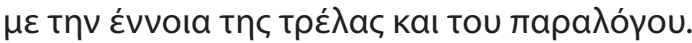

\section{Mпретóv: Проброникótпта}

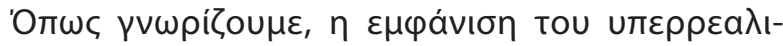

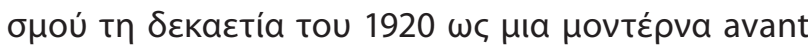

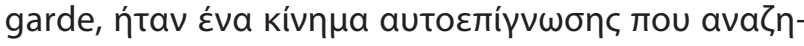

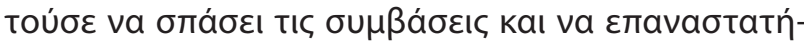

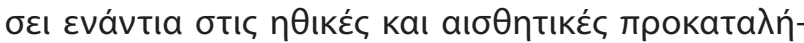

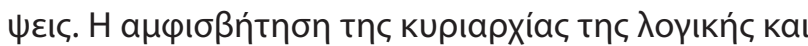

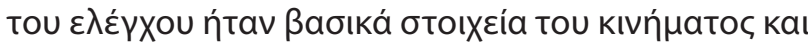

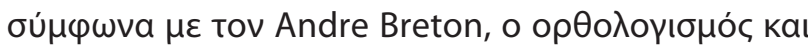

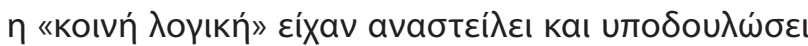

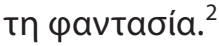

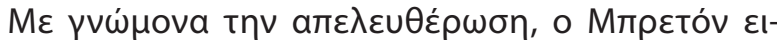

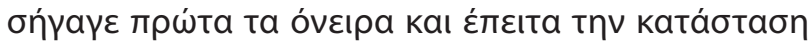

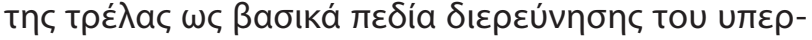

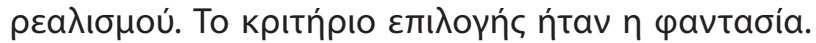

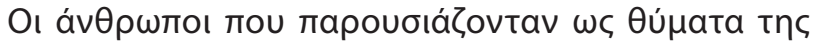

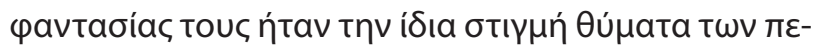

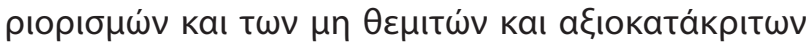

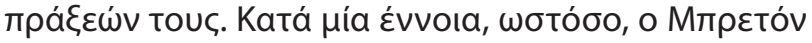

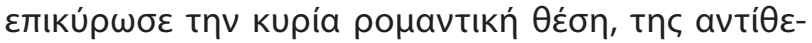

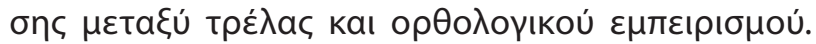

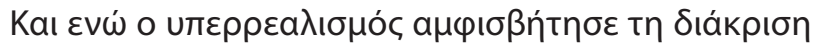

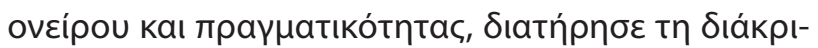

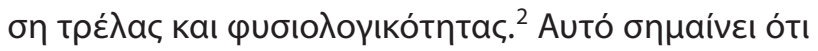

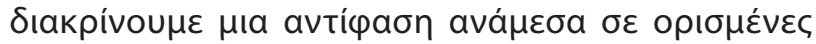

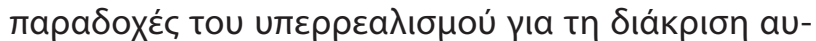

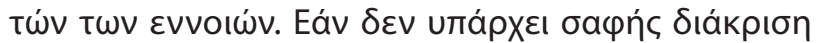

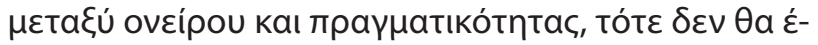

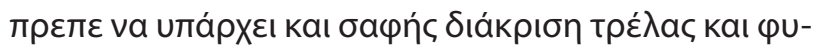

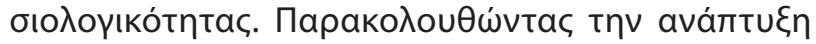

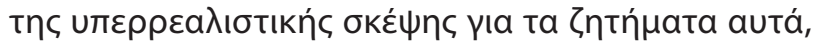

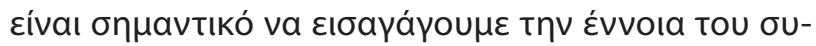

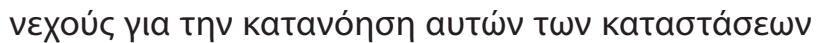

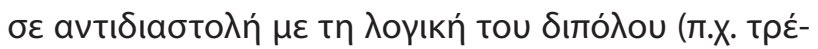
$\lambda a-\varphi$

\section{Ntaลí: $\Delta$ optкótnta}

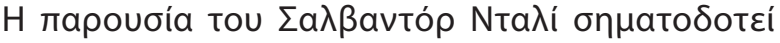

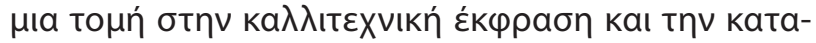

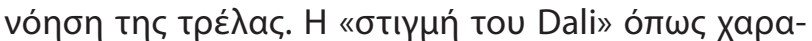

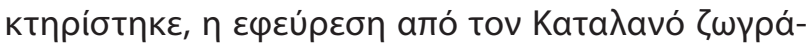

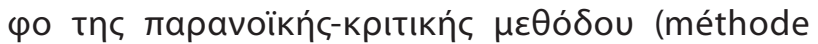

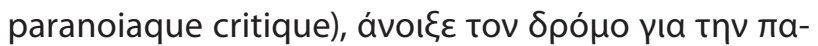

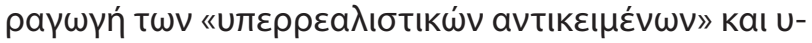

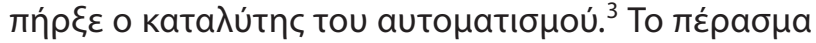

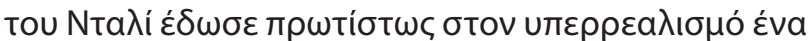

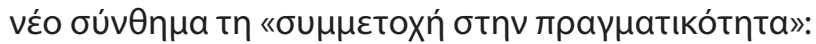

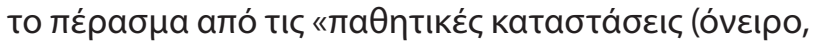

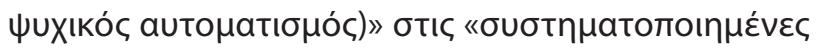

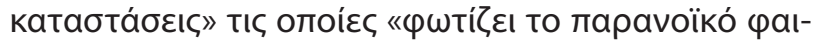
vó $\mu \varepsilon v 0 » .{ }^{9,10}$

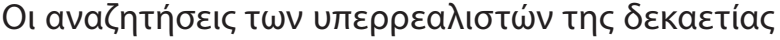

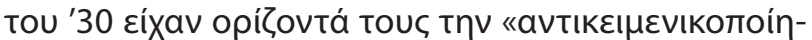

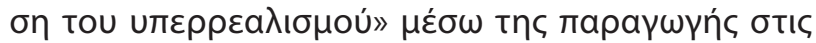

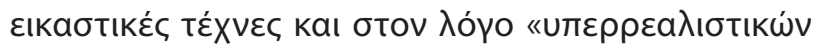

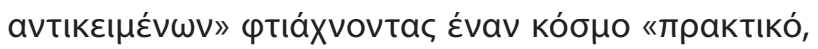

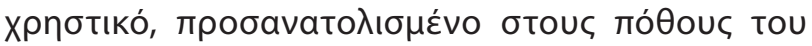

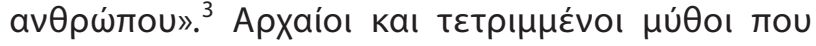

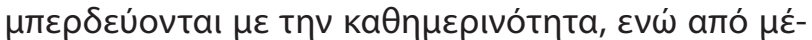

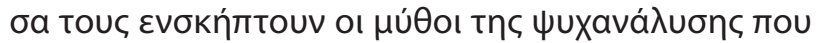




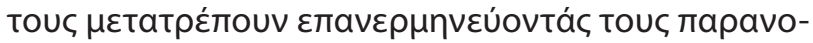

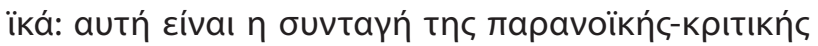

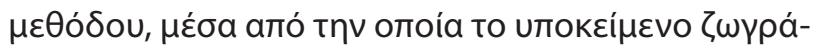

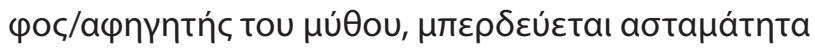

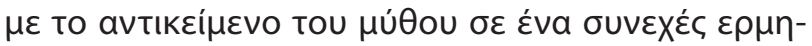

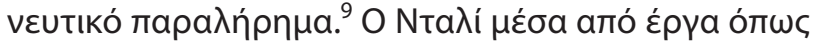

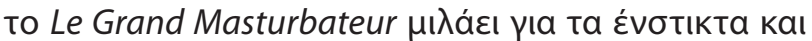

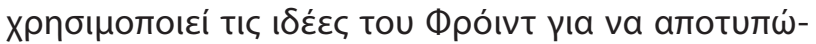

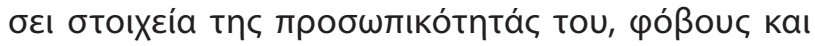

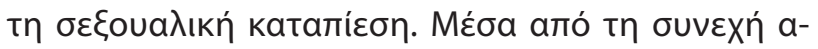

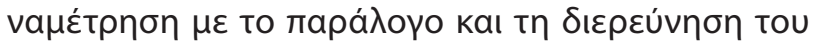

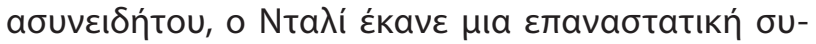

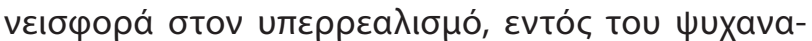

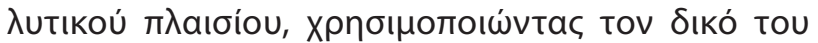

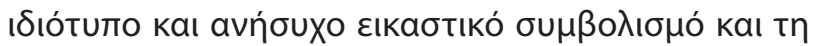

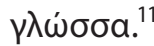

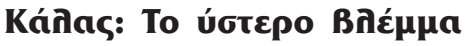

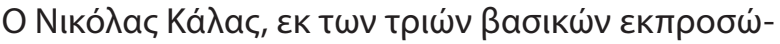

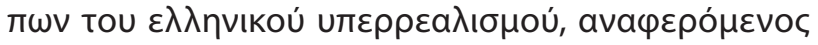

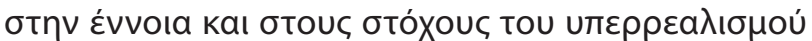

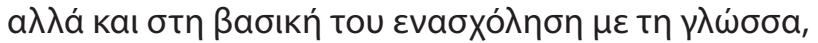

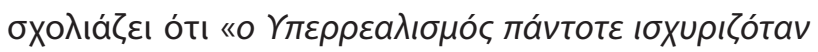
ótı

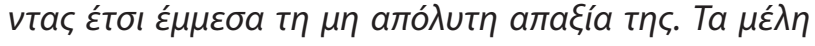

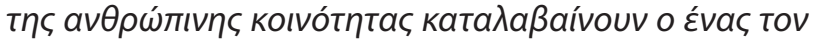

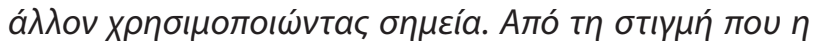

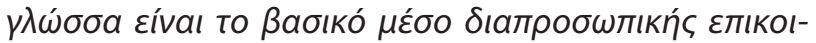

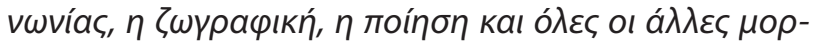

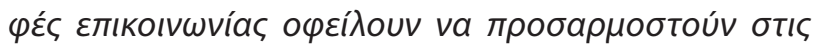

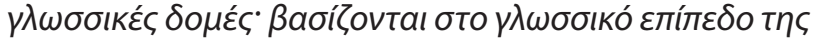

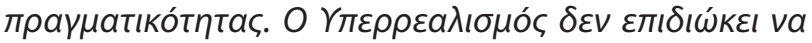

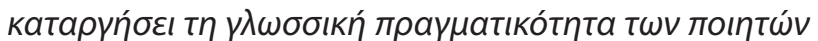

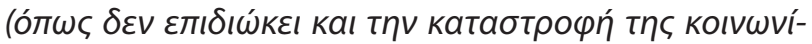

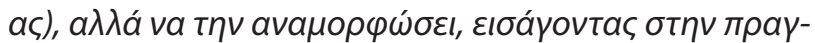

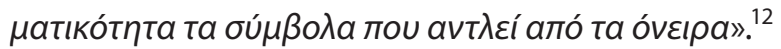

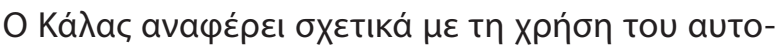

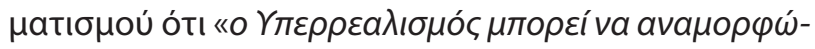

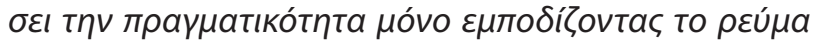

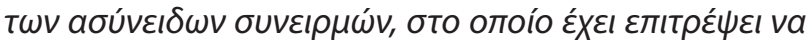

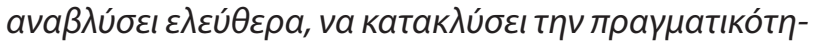

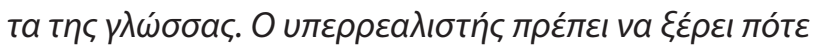

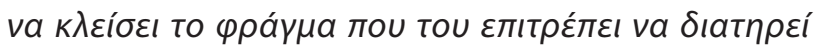

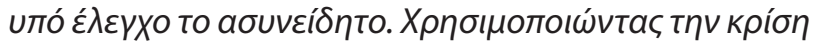

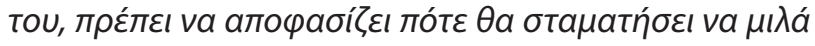

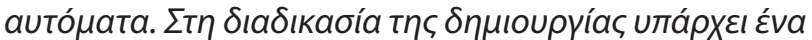

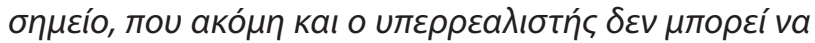

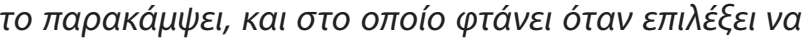

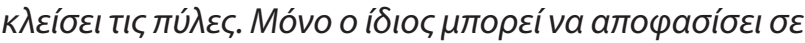

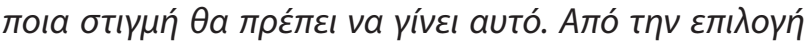

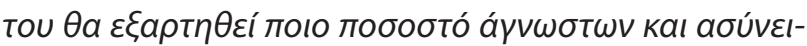

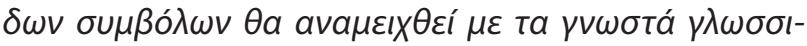

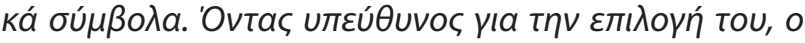

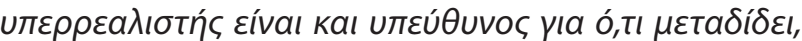
a

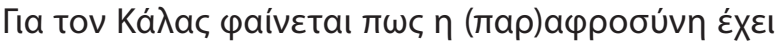

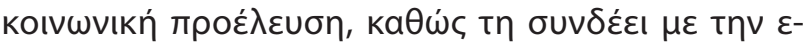

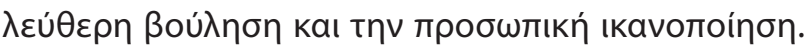

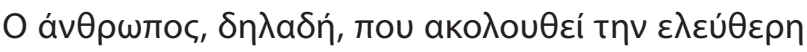

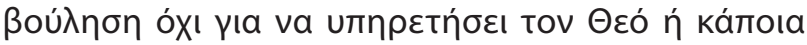

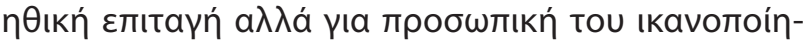

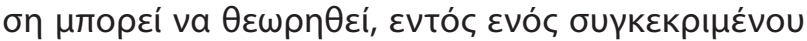

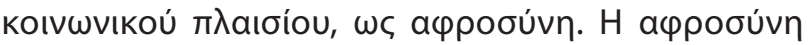

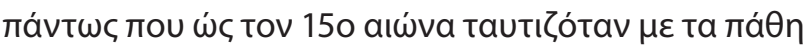

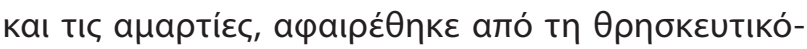

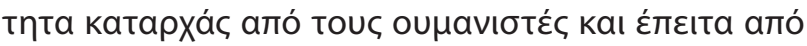

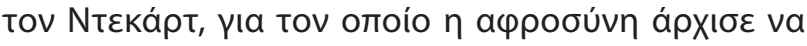

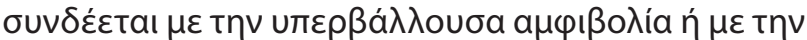

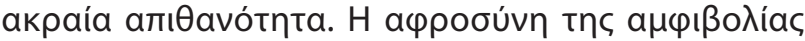

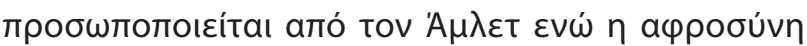

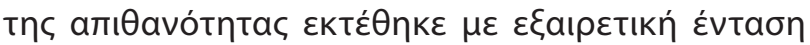

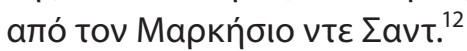

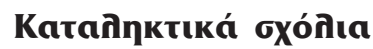

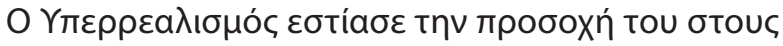

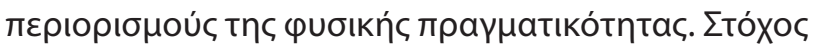

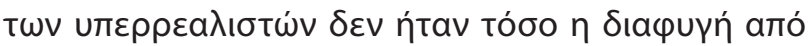

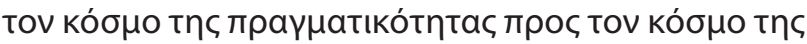

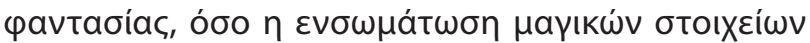

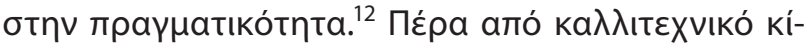

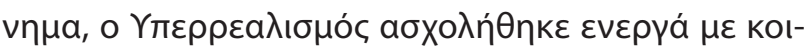

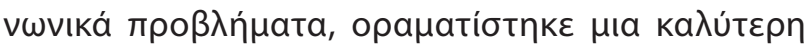

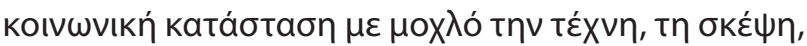

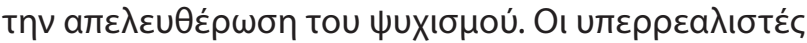

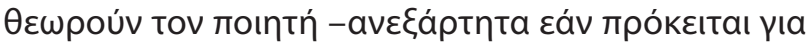

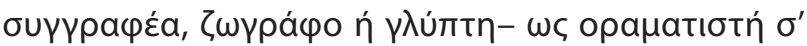

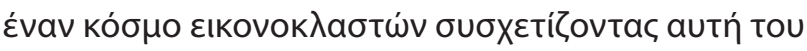

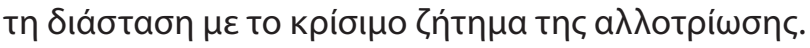

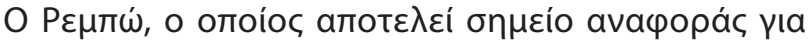

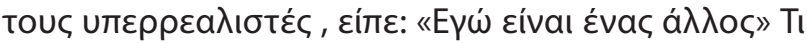

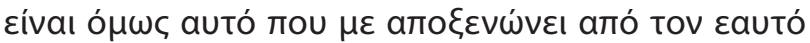
$\mu \mathrm{ov}{ }^{12}$

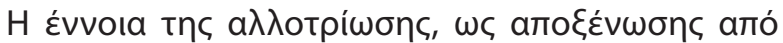

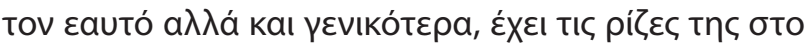




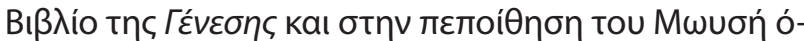

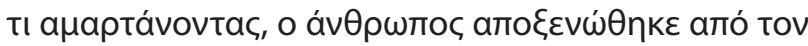

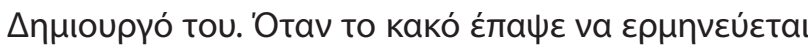

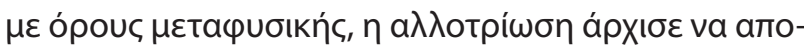

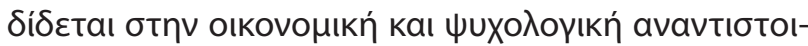

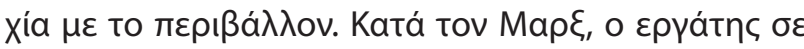

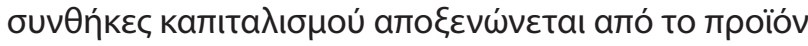

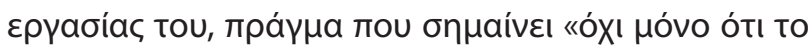

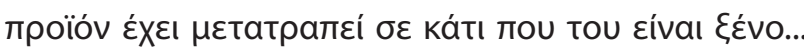

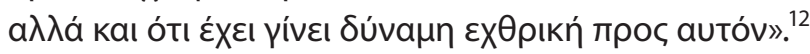

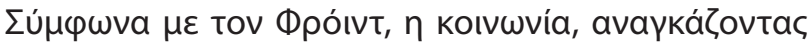
tov ávӨ

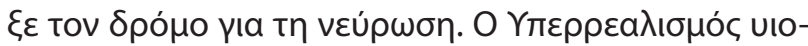

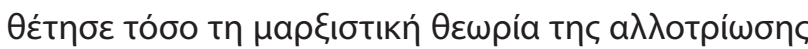

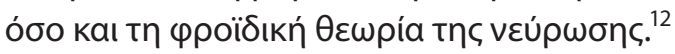

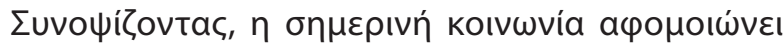

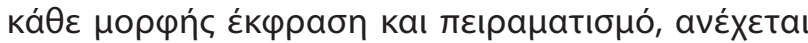

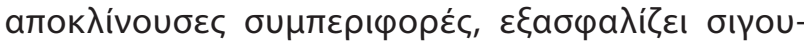

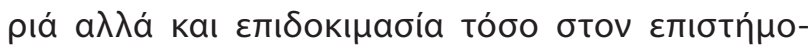

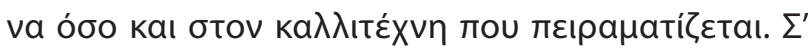

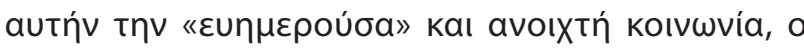

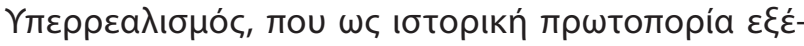

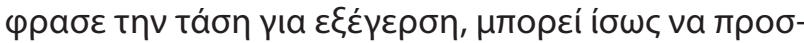

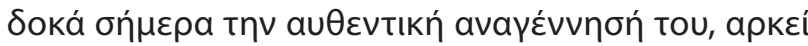

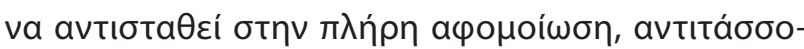

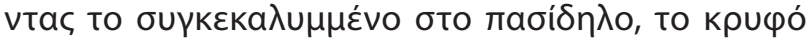

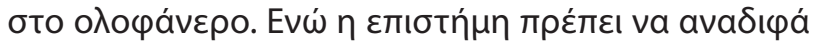

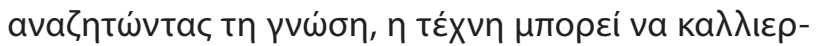

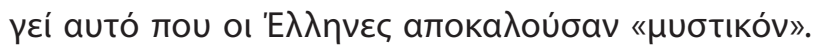

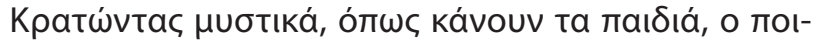
птท́

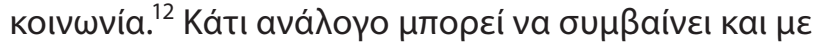

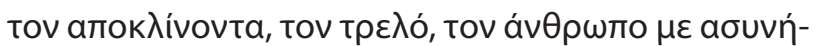

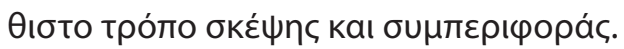

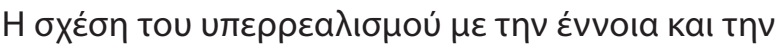

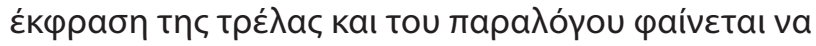

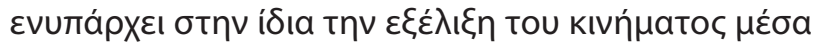

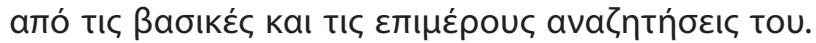

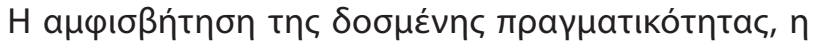

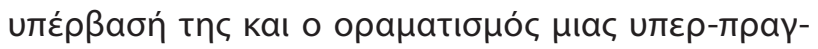

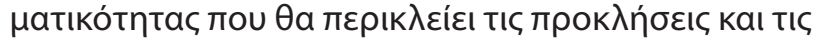

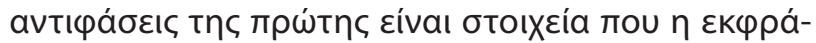

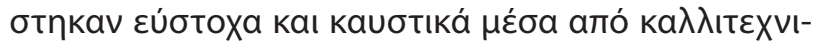

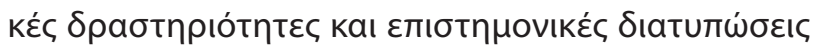

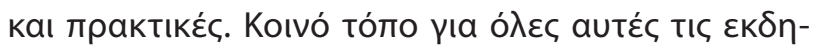

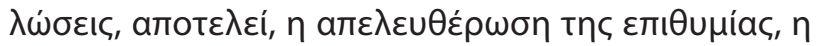

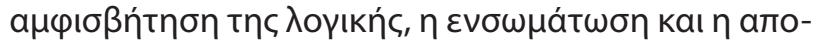

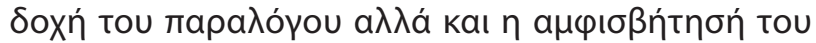

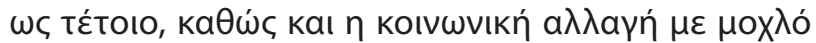

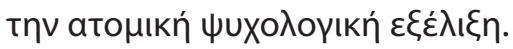

\title{
Surrealism and madness
}

\author{
K. Flora \\ University of Neapolis, Pafos, Cyprus, \\ Aristotelian University of Thessaloniki, Thessaloniki, Greece
}

Psychiatriki 2017, 28:360-366

This article attempts an approach of madness by surrealism, as reflected in the pathway of the surrealist movement. In the light of enlargement of the concept of mental illness and the experience of madness, an approach is being attempted regarding the early surrealist views as they precursory appear e.g. from the case of Hieronymus Bosch to the meeting of the dominant psychiatry and the surrealist movement in the 19th and 20th century. Then, the paper attempts to present the main positions of representatives of the movement, such as Breton, Dali and Kalas. These three surrealists were chosen among others, for this brief report, as the representatives of three remarkable moments in the surrealistic route. Breton introduces the element of fiction and hyper-reality while he questions the distinction between normal and abnormal element. Dali 
with his paranoid critical method reconciles actual representations with mythical and symbolic elements, breaking through the limits between objectivity and subjectivity. Kalas puts forward the social origin of insanity along with the fundamental surrealist notions of individual freedom and will. For a more complete understanding of this attempt, it was considered useful to include elements of the main views on madness from the standpoint of a critical approach in psychiatry and psychology. The surrealistic view seems to be close to this critical approach which is likely to have been affected by it on the level in which the movements and scientific fields meet and interact. The relationship between surrealism, the notion and expression of madness and the absurd seems to be inherent to the very development of the movement through its core and individual pursuits. In conclusion, the relationship between surrealism and the notion and expression of the madness and the absurd seems to be inherent to the very birth of the movement through its main positions and pursuits. The question of so-called reality, its overshoot and the vision of a hyperreality that will incorporates the challenges and contradictions of this reality, are points loudly expressed through artistic activities and scientific researches and practices. A common ground to all these aspects is the liberation of desire, the questioning of rationality, the integration and acceptance of the absurd, as well as, the dispute of it as such, and finally the social change-driven by the individual psychological development.

Key words: Surrealism, insanity, absurd, representatives.

\section{BıBลtoypacpía}

1. Gross CG. "Psychosurgery" in Renaissance art. Trends Neurosci 1999, 22:429-431, PMID: 10481185

2. Constantinidou D. The paranoid simulacrum in surrealism: From embracing madness to the mechanism of a mental Illness as the purveyor of individual meaning. Gramma 2010, 18:119-133

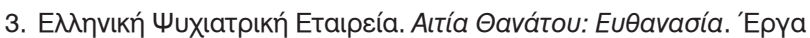

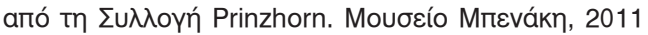

4. Davies D. On the very idea of "outsider art". Br J Aesthet 2009, 49:25-45, doi:10.1093/aesthj/ayn056

5. Rhodes, C. Outsider art: Spontaneous alternatives. Thames and Hudson, London, 2000

6. Peiry L. Art Brut: The origins of outsider art. Frank J (transl), Flammarion, Paris, 2001

7. Rabaté JM, Loving Freud madly: Surrealism between hysterical and paranoid modernism. J Mod Literat 2002, 25:58-74, doi: 10.1353/jml.2003.0023

8. Breton A. Communicating Vessels. Caws MA, Harris GT (transl), University of Nebraska Press, 1990, p. 11 [See also Les vases Communicants I, in André Breton, Oeuvres Complètes II. Bonnet M, Bernier P, Hubert E-A, Pierre J (eds) Gallimard, Pléiade, Paris, 1992, p.152]

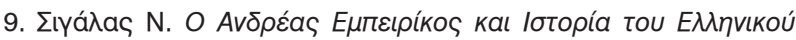

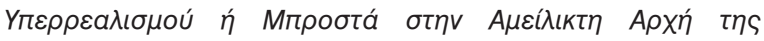

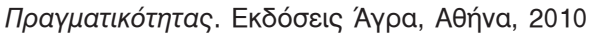

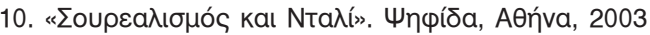

11. Herrera MJ, Alcantara AG, Garcia F. Dali (1904-1989): Psychoanalysis and pictorial surrealism. Am J Psychiatry 2003, 160:855856, doi:10.1176/appi.ajp.160.5.855

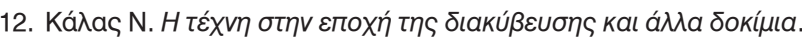

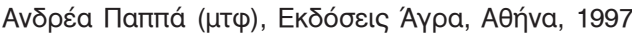

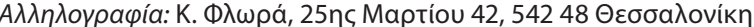
$\operatorname{T\eta \lambda .:} 6973238267$

e-mail: katerinaflora@hotmail.com 